\section{Mixed signals in heart failure: cancer rules}

\author{
Masahiko Hoshijima and Kenneth R. Chien
}

University of California, San Diego (UCSD) Institute of Molecular Medicine, UCSD-Salk Program in Molecular Medicine, La Jolla, California, USA

Address correspondence to: Kenneth R. Chien, UCSD Medicine 0641, 9500 Gilman Drive, La Jolla, California 92093, USA.

Phone: (858) 534-6835; Fax: (858) 534-8081; E-mail: kchien@ucsd.edu.

J. Clin. Invest. 109:849-855 (2002). DOI:10.1172/JCI200215380.

cancer: a disease marked by tumor-like growth -Merriam-Webster's Ninth Collegiate Dictionary

In the world according to Webster, heart failure is a form of cardiac cancer. Although primary cardiac malignancies are among the most rare of human diseases, this singular viewpoint is substantiated by the massive, abnormal, "tumor-like" growth in cardiac muscle that accompanies heart failure. The biological principles of cell growth, death, and survival are as important in the onset of heart failure as in tumor progression, and the molecular signals for cell proliferation and cardiac myocyte hypertrophy are highly conserved. Both diseases are inexorable and progressive, characterized by clinical stages that predict survival and outcome, ultimately resulting in a terminal phase. There are "multihit" pathways for both cancer and heart failure progression, largely based upon the interplay between genetic susceptibility and environmental stimuli. Like cancer, heart failure represents one of the most important unmet clinical needs in medicine today.

Heart failure remains poorly understood and is largely treated symptomatically by a complex regimen of drugs whose actions we are neither entirely sure of nor comfortable with. The lack of new biologically targeted therapy reflects the combined result of the prohibitive cost of large-scale survival trials of adjunctive therapy designed to incrementally slow heart failure progression, and the lack of clear clinical and/or molecular surrogates that have predictive value. In short, the pipeline of heart failure drugs is running dry.

Perhaps the time has come to attempt to dissect the mixed signals for heart failure from the viewpoint of recent advances in cancer biology. On a molecular level, the failing heart represents the end result of multiple cues for the growth, death, and survival of cardiac myocytes, many of which are shared with signaling pathways in cancer biology $(1,2)$. In the failing heart, the underlying disease is also driven by multiple posi- tive and negative signaling pathways, but these are connected to specific phenotypic endpoints that are more physiologically complex, e.g., electrical conduction, contractility, relaxation, mechanical activation, and chamber morphogenesis (Figure 1). The development of new, biologically targeted therapies for cancer has been fostered by placing therapy into the framework of fundamental pathways that control the cell cycle and subsequent tumor cell proliferation. In a similar manner, understanding the molecular logic of heart failure will ultimately require forming mechanistic connections between defined clinical disease surrogates with specific positive and negative molecular checkpoints that arise at specific stages during the natural temporal progression of this chronic disease. In the current post-genome world, a new wave of work in heart failure has begun to attack the integrative biology of heart failure at multiple levels: genomic, genetic, and physiological in creatures great and small (3). This brief review will highlight recent progress in the field and point out new directions for research into this disease process.

\section{Biphasic temporal signaling cascades in heart failure progression}

Stretch activation response: positive and negative regulators of mechanical growth and survival cues. Mechanical stress is one of the most important stimuli for triggering the initial steps toward heart failure (4). A diverse group of biochemical signals are rapidly activated during in vivo pressure overload, many within a few minutes of exposure to dynamic or passive stretch, increased aortic pressure, or volume overload. As part of this acute response, peaking within hours following the introduction of an aortic banding, a host of downstream cytoplasmic kinases are activated, including Akt and the ERKs, JNKs, p38s, and Janus kinases (JAKs) $(5,6)$. These kinases have all been implicated in the subsequent steps of activation of either myocyte survival or growth responses (Figure 1). Interestingly, a systematic temporal analysis reveals a double peak of activation for many of these downstream kinases (5), suggesting that there may be discrete roles for some of these pathways in the activation phase and in the hypertrophic phase, which follows over the next several days $(4,7)$. However, work in the various genetically engineered mouse models developed to study these regulatory proteins has generally not addressed specific temporal requirements in these two distinct phases. Indeed, it may be necessary to design genetically manipulable systems that will allow a careful observation of the sequential activation of signaling cascades in the initial stretch activation response of intact cardiac muscle. At the core of this problem will be the identification of the molecular nature of the stretch sensor/activator complex and understanding how this triggers specific molecular signals. Currently, little is 


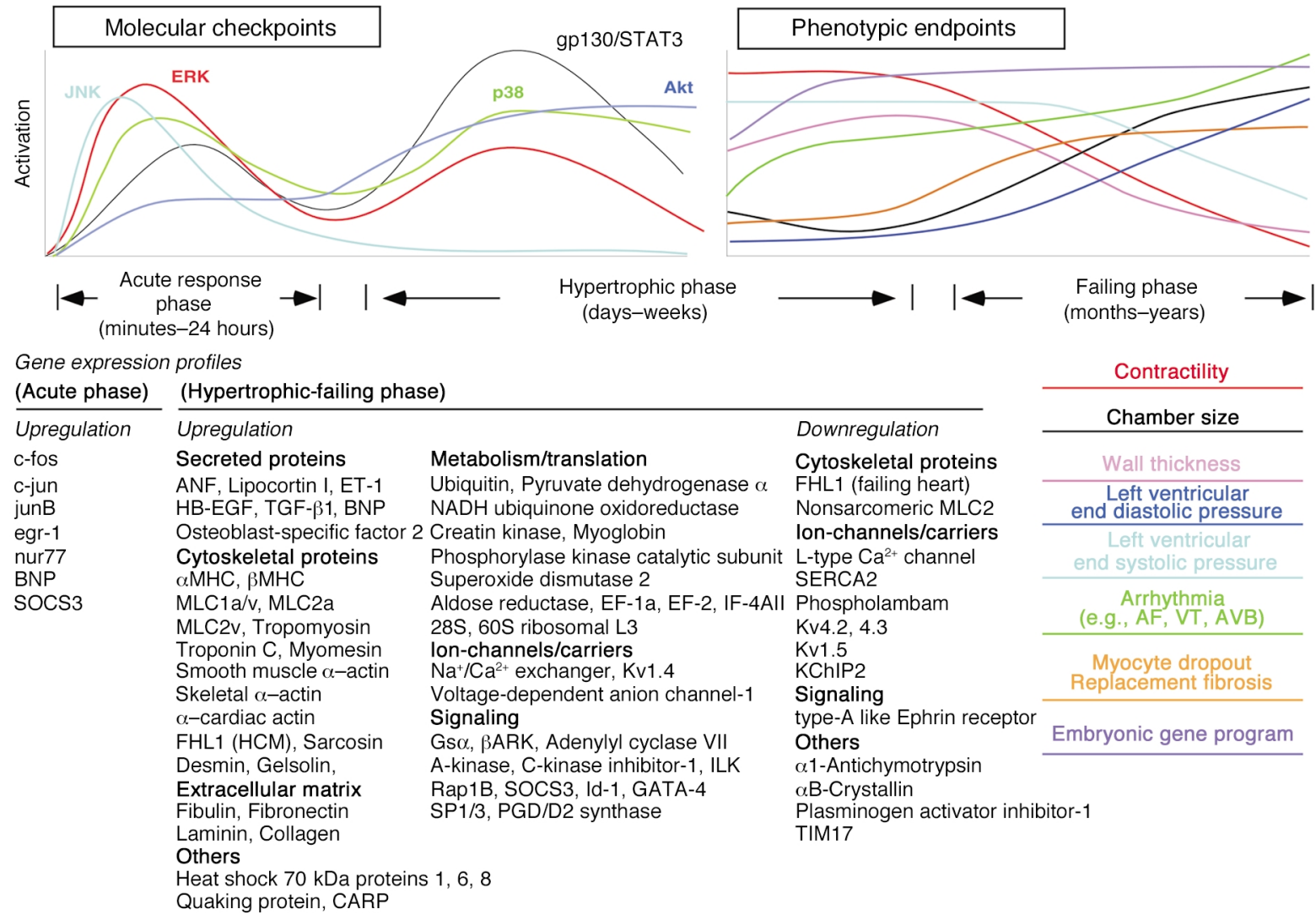

Figure 1

Mechanical stress-induced transmembrane signaling and integrated phenotypes of failing heart. The activation profiles of various signaling cascades and time-dependent changes in phenotypic features of heart failure are illustrated based on our previous studies (5) and unpublished observations. The lower panel shows the expression profiles of selected genes from publications in both human and animal studies. See supplemental reading list (www.jci.org/cgi/content/full/109/7/849/DC1) for detailed references.

known regarding whether the stretch sensor/activator represents a defined function of a new class of stretch receptors or stretch-activated channels, or a macromolecular complex within the intracellular cytoskeleton, or perhaps a complex of ECM components.

The double peak of early stretch activation responses implicates the presence of negative regulators that act as a negative feedback loop to prevent the continuous activation of any single pathway. Studies in transgenic mice now clearly document that the sustained activation of almost any individual signaling molecule may cause deleterious effects on cardiac function and promote secondary hypertrophy. Accordingly, there must be negative regulators of the response that attenuate the signal so that an orderly compensatory hypertrophic response ensues, rather than unbridled cardiac enlargement. Although numerous studies have suggested essential roles for a variety of intracellular signaling pathways in the regulation of cardiac hypertrophy and failure $(1,7)$, a growing number of studies have identified negative feedback regulation of these intracellular signaling pathways. In this regard, suppressor of cytokine signaling 3 (SOCS3) provides an example of a biomechanical stress-inducible negative regulator that inhibits the gp130 stress-inducible pathway for myocyte survival (5).
Within minutes of aortic constriction, gp130 ligands such as cardiotrophin-1 and leukemia inhibitory factor activate JAKs, leading to the phosphorylation of STAT3. STAT3 translocates into the nucleus and directly activates the expression of BCL- $\mathrm{X}_{\mathrm{L}}$ and VEGF and other factors that regulate myocyte survival and cardiac remodeling (8). Among these, SOCS3 is induced in the myocardium within 1 hour of aortic banding and peaks after a couple of hours. By inhibiting JAKs, SOCS3 negatively regulates stress-inducible gp130 activation. In this manner, the delicate balance between the activation of gp130-JAK signaling and the induction of its negative feedback regulator SOCS3 might be important in the transition between cardiac hypertrophy and failure, via attenuation of myocyte survival signals. Disrupting the balance between this positive and negative regulatory loop might lead to secondary effects that impair cardiac function. Accordingly, to promote gp130 myocyte survival pathways, it might be helpful to design inhibitors of the negative regulator, as opposed to designing ways to constitutively activate gp130 pathways, an event that may excessively stimulate downstream signalings. Cardiac-specific inducible mutagenesis of SOCS3 within a specific temporal period following in vivo pressure overload should be informative with regard to the validity of this new therapeutic strategy. 
A number of other novel negative regulators of cardiac signaling have recently been described. Recent studies have identified myocyte-enriched calcineurin-interacting protein-1 (MCIP1) as a protein that inhibits in vivo cardiac hypertrophy by attenuating calcineurin activity (9). The MCIP1 gene contains an intragenic calcineurin response element with a cluster of NF-AT transcriptional factor binding sites, thereby suggesting that MCIP1 participates in the negative feedback loop of calcineurin signaling in myocardium (10). Bueno et al. reported a negative feedback system for the cardiac mitogen-activated protein (MAP) kinase signaling cascade in which forced expression of MAP kinase phosphatase-1 negatively regulates the cardiac hypertrophic response by downregulating p38, JNK-1/2, and ERK-1/2, which define the three branches of the MAP kinase pathway (11). As there must be endogenous negative regulators that titrate the signals derived from the other stretchactivated cytoplasmic kinase-dependent pathways, it will be of interest to develop assays to identify suppressors of specific steps of cardiac signaling during stretch activation responses.

The bypertrophic response: quantitative effects of increasing translational efficiency and qualitative effects on the induction of an embryonic gene program. Cardiac hypertrophy can be broken down into two central phenotypic endpoints: quantitative effects on increasing the level of sarcomeric and other constitutive proteins, and qualitative changes in the cardiac gene program that lead to a return to the fetal phenotype. Interestingly, both of these phenotypic effects can be found during growth stimulation of diverse differentiated cell types. In this regard, IGF-1 is a potent stimulus for both cardiac and skeletal muscle hypertrophy in vivo. Using IGF-1 as a model in vitro growth stimulus, recent studies have shown that an increase in translational efficiency of pre-existing mRNAs underlies the hypertrophic response of skeletal muscle hypertrophy. A number of independent approaches have convincingly shown a central role of Akt in enhancing translational efficiency by activating mammalian target of rapamycin (mTOR) or phosphorylating and thereby inhibiting glycogen synthase kinase 3 (GSK3) $(12,13)$. Muscle atrophy leads to a decrease in the Akt/mTOR pathway, and IGF-1 unexpectedly acts via Akt to antagonize calcineurin signaling during myotube hypertrophy. Taken together, the activation of the Akt/mTOR pathway and its downstream targets, p70S6 kinase, PHAS-1/4E-BP1, and GSK3, appears to play a central role in skeletal muscle hypertrophy and atrophy. An analogous increase in translational efficiency occurs during in vivo pressure overload cardiac hypertrophy. Moreover, myocardial Akt is activated early during both the acute phase and the hypertrophic phase (Figure 1) $(5,14)$. Taken together, a similar role for Akt signaling in the hypertrophied heart is likely.

In contrast to skeletal muscle hypertrophy, studies in cardiac muscle now support an important role for calcium signaling in the activation of a hypertrophic response. Dual activation of calcium/calmodulindependent kinase and calcineurin-dependent pathways has been documented in vivo (15), and recent studies employing cardiac-specific expression of a dominant negative form of calcineurin (16), endogenous calcineurin inhibitors $(9,17)$, have documented a pivotal role for this pathway in vitro and in vivo. However, a number of other proximal signals have also been shown to be both necessary and sufficient to activate a cardiac hypertrophic response in vitro and in vivo (7). A growing body of clinical and scientific evidence now supports the view that $G_{q} / G_{11}$ signaling acts at one of the proximal steps in the in vivo hypertrophic response. Mice harboring a double cardiac-restricted ablation of both $G \alpha_{q}$ and $\mathrm{G} \alpha_{11}$ display a complete loss of in vivo pressure overload hypertrophy (18), whereas partial inhibition of the hypertrophic response has been noted in studies examining the loss of function of other signaling molecules. It will be of interest to determine the extent of cross-talk between calcium and $G_{q} / G_{11}$ pathways and to identify their differential role in triggering downstream phenotypic features of cardiac hypertrophy and failure. Of particular interest will be the identification of the compartmental source of calcium that triggers biochemical calcium-dependent signaling as well as the pool of calcium that contributes to transient increases in calcium and allows for excitation-contraction coupling.

The activation of a fetal gene program is a highly conserved feature of the hypertrophic response and has been extensively studied as a means to identify physiological regulators of hypertrophy (Figure 1). Although the initial steps of the induction of embryonic genes are clearly reversible, chronic changes in the cardiac gene program may trigger pathological changes in the myocardium that can be associated with irreversible dysfunction. Much of this work has focused on the inducibility of the atrial natriuretic factor $(A N F)$ gene, which is reactivated in ventricular muscle early during the hypertrophic response. A host of cardiac transcription factors work combinatorially to control the expression of ANF and a panel of downstream targets in the embryonic gene program, including the genes for Nkx2.5, the GATA factors, NF-AT, Tbx5, and MEF2 (19-23). The recent discovery of a new series of striated muscle-restricted transcriptional cofactors suggests new combinatorial pathways by which specific sets of cardiac/skeletal muscle-specific genes, such as HDAC and Myocardin, can be selectively regulated in response to defined upstream signals $(15,24)$. The growing list of target genes that are downregulated during this process includes some that underlie specific surrogate disease endpoints, such as decreases in calcium cycling, conduction defects, and channel genes and regulators that can create an electrophysiological substrate for arrhythmogenesis (Figure 1). It will be of interest to determine whether the differential regulation of this subset of negatively regulated cardiac restricted genes reflects the effects of distinct ligands or downstream transcription factors that account for their counter-regulation.

\section{Pathways for surrogate failing heart phenotypes}

Chamber dilation: diverse cytoskeletal pathways. Changes in organ size, shape, and histological phenotype are central features of both tumorigenesis and heart failure. Chronic increases in ventricular volume and pressure 
Table 1

Molecular defects linked to dilated cardiomyopathy

\begin{tabular}{|c|c|c|}
\hline Genomic defects & Defects in humans & Animal models \\
\hline \multicolumn{3}{|l|}{ Sarcomere } \\
\hline $\begin{array}{l}\text { Myosin heavy chain } \\
\text { Cardiac actin } \\
\text { Troponin-T }\end{array}$ & $\begin{array}{l}\text { Missense } \\
\text { Missense } \\
\text { Deletion }\end{array}$ & \\
\hline \multicolumn{3}{|c|}{ Titin/titin-related protein } \\
\hline Titin & Missense/Deletion & CM zebra fish \\
\hline \multicolumn{3}{|c|}{ Z-disk-associated proteins } \\
\hline $\begin{array}{l}\text { MLP } \\
\text { ALP } \\
\text { ZASP/Cypher }\end{array}$ & & $\begin{array}{c}\mathrm{DCM}(\mathrm{KO}) \\
\text { RV-dominant CM (KO) } \\
\text { Congenital CM (KO) }\end{array}$ \\
\hline \multicolumn{3}{|c|}{ Sarcolemma cytoskeleton } \\
\hline $\begin{array}{l}\text { Dystrophin } \\
\gamma \text {-Sarcoglycan } \\
\delta \text {-Sarcoglycan }\end{array}$ & $\begin{array}{l}\text { Deletion } \\
\text { Missense }\end{array}$ & $\begin{array}{c}\text { CM (utrophin/dystrophin double KO) } \\
\text { Atypical CM (KO) } \\
\text { CM (KO, vascular involvement) } \\
\text { CM hamsters }\end{array}$ \\
\hline$\alpha$-Dystrobrevin & Missense & Mild CM $(K O)$ \\
\hline Metavinculin & Missense/Deletion & \\
\hline \multicolumn{3}{|c|}{ Intermediate filaments } \\
\hline $\begin{array}{l}\text { Desmin } \\
\text { Lamin A/C }\end{array}$ & $\begin{array}{l}\text { Missense } \\
\text { Missense }\end{array}$ & $\mathrm{CM}$ with calcification $(\mathrm{KO})$ \\
\hline
\end{tabular}

MLP, muscle-specific LIM-only protein; ALP, $\alpha$-actinin-associated LIM protein, ZASP, Z-band alternatively spliced PDZ motif protein; CM, cardiomyopathy; DCM, dilated CM; KO, knockout mouse. See supplemental reading list (www.jci.org/cgi/content/full/109/7/849/DC1) for detailed references.

higher throughput strategies for clinical genotyping. It will also be of particular interest to determine whether cytoskeletal mutations have regulatory effects on specific intracellular signaling pathways triggered by biomechanical or hormonal stimuli, which would imply a more direct effect on heart failure progression. If dysregulation of cytoskeletal signaling can be shown to trigger chamber dilation in acquired forms of heart failure, it may indeed be possible to generalize from observations made with rare genetic forms of dilated cardiomyopathy to more common forms of the disease. The recent association of cytoskeletal components with a distinct component of cardiac channels ( $\mathrm{minK}$ ) raises the intriguing possibility that key cytoskeletal components directly regulate cardiac channels involved in control of cardiac repolarization and/or excitation-contraction coupling (25).

Contractility and relaxation defects: compartmental control of cardiac signaling and calcium cycling. During the course of cardiac chamber dilation, a progressive decrease in cardiac contractility and relaxation ensues, which ultimately leads to chronic heart failure. The precise link between chamber dila-

can lead to dilation of the cardiac chamber and subsequent thinning of the ventricular wall during advanced heart failure. Diverse cytoskeletal mutations have recently been associated with dilated cardiomyopathy in both experimental and clinical studies (Table 1). Based on their specific locations within the cardiomyocyte cytoskeletal network, distinct classes of cytoskeletal defects have been uncovered that include components of the macromolecular titin complex, Z-disk and intercalated disc proteins, components of the dystrophin/dystroglycan complex, and mutations in the nuclear and intermediate filaments. Since the cytoskeleton serves multiple functions in the cardiac myocyte, a number of theories have been proposed that might link the various cytoskeletal defects with chamber dilation, including defects in force transmission, force generation, sarcolemmal integrity, sarcomeric organization/assembly, and intercalated disc stability.

How well these genetically based forms of chamber dilation reflect events in the more prevalent form of heat failure, which occurs many months after myocardial infarction, remains an open question. Since a diverse group of cytoskeletal mutations have now been linked with chamber dilation, a clinical issue of immediate interest will be to determine whether heterozygous mutations or functionally important polymorphisms in cytoskeletal genes might confer an increased risk for a more rapid onset of heart failure progression during cardiac remodeling in response to acquired forms of cardiac injury. The growing number of known and novel genes that are causally related to chamber dilation, and the vast genotypic heterogeneity within any given cytoskeletal disease gene, underscore the need for tion and cardiac dysfunction is not completely clear, as there are numerous defects that have been associated with the impairment of cardiac contractile function in heart failure. However, numerous studies point to a central role of defects in calcium cycling that arise from a decrease in the activity of the sarcoplasmic reticulum calcium ATPase (SERCA), which regulates diastolic and systolic function via the re-sequestration of calcium into the internal sarcoplasmic reticulum (SR) pool (Figure 2) (26-28). During the onset of heart failure, the decrease in SERCA activity largely relates to the unrestrained inhibitory effect of an endogenous muscle-specific SERCA-inhibitory peptide, phospholamban. In the normal heart, phospholamban's inhibitory activity is under the control of the $\beta$-adrenergic receptor-adenylyl cyclase-protein kinase A pathway, whereby phosphorylation of the Ser16 residue of this peptide suppresses the peptide's inhibitory effect. In this manner, one of the critical downstream targets in the cAMP-dependent pathway for enhancing cardiac contractility and relaxation is relieving SERCA from phospholamban inhibition via phosphorylation. In human and experimental models of heart failure, phospholamban is hypophosphorylated $(29,30)$, which most likely reflects desensitization of the $\beta$-adrenergic pathway due to the increase in the activity of $\mathrm{G}$ protein-coupled receptor kinases (GRKs), including the $\beta$-adrenergic receptor kinase (BARK/GRK2), in the failing heart (31).

Although clinical and experimental studies document that simply enhancing cAMP during heart failure can lead to cardiac injury and an associated increase in mortality, recent studies have identified new therapeutic targets in the molecular pathways that control car- 
diac contractility and relaxation (Figure 2 ). The clear beneficial effects of $\beta$-blockade on survival in clinical heart failure suggest that any new therapy will most likely need to be compatible with chronic $\beta$-blocker therapy. In this regard, genetic complementation studies and somatic gene transfer in acquired forms of heart failure have now suggested that it is possible to markedly improve cardiac diastolic and systolic function independent of effects on cAMP levels by enhancing calcium cycling (32). Relieving the desensitization of the $\beta$-adrenergic receptor via GRK inhibition has also been shown to ameliorate the progression of heart failure and promote survival in experimental models (31). It will be of particular interest to determine whether additional cardiac receptors escape desensitization and can therefore activate calcium cycling and promote cardiac contractility following heart failure. New insights into the effects of altered calcium release channels (33) suggest novel therapeutic targets within specific intracellular compartments such as the SR, the T-tubular apparatus, and functional/structural microdomains of the cardiac sarcolemma.

Arrbythmogenesis: acquired defects in conduction lineages, intercellular coupling, and channel modulators. One of the major causes of mortality in heart failure is the onset of life-threatening malignant arrhythmias. The incidence of cardiac sudden death and associated arrhythmogenesis correlates with the severity of dysfunction, but the mechanisms that account for this markedly increased susceptibility in the failing heart are unclear. A growing body of electrophysiological, genetic, experimental, and clinical data now supports a multi-hit mechanism for arrhythmogenesis $(34,35)$. In addition to the enhanced secondary impulse of afterdepolizations, the heterogeneity of action potential duration throughout the myocardium of the failing heart may account for the arrhythmogenic substrate that creates susceptibility for re-entrant ventricular tachyarrhythmias.

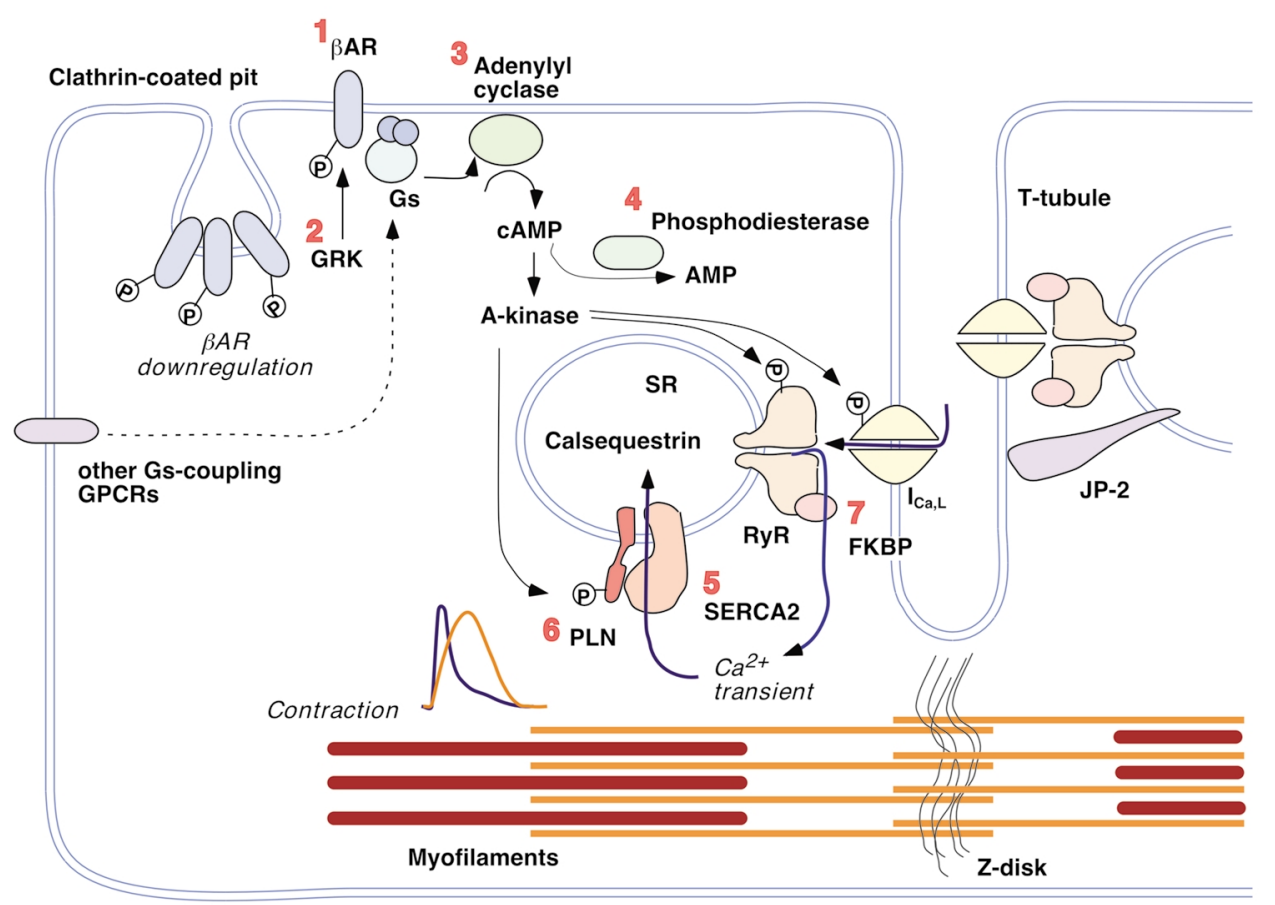

\section{Figure 2}

New therapeutic targets of heart failure in regulatory pathways of excitation-contraction coupling. Excitation-contraction coupling and its regulation by Gs-activating G protein-coupling receptor (GPCR) and A-kinase signaling. A series of genes have been subjected to target validation analysis via transgenic and somatic gene transfer studies with a variety of viral vector systems and protocols. 1. $\beta 2$-adrenergic receptor $(\beta 2 A R)$. $\beta 2 A R$ transgenic $(\beta 2 A R-T G)$ mice display a hypertrophic cardiomyopathy phenotype and do not rescue the phenotype of failing muscle-specific LIM-only protein knockout (MLPKO) mice. $\beta 2 A R-T G$ mice fail to rescue the HCM phenotype of R403Q $\alpha$ MHC-TG mice. Percutaneous or intramuscular Adeno/ $\beta 2 A R$ delivery results in enhanced contractility in normal rabbit or cardiomyopathic hamsters. 2. $\beta A R K$ inhibitor ( $\beta$ ARKct). $\beta A R K c t-T G$ mice partially rescue cardiomyopathic phenotypes of

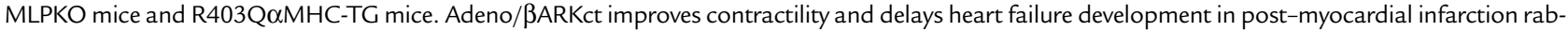
bits. 3. Adenylyl cyclase type VI (AC-typeVI). AC-typeVI-TG mice rescue heart failure in $\mathrm{G}_{\mathrm{q}}$-TG mice. Percutaneous Adeno/AC-typeVI delivery enhances cardiac contractility in normal pigs. 4. Phosphodiesterase. Several human clinical trials have confirmed that phosphodiesterase's pharmacological inhibition improves cardiac function, although at the risk of decreased long-term survival; current clinical use is primarily confined to end-stage heart failure awaiting transplantation. 5. SERCA2: SERCA2-TG mice are hyperkinetic, whereas heterozygous null animals are hypokinetic. Adeno/SERCA2 gene delivery improves cardiac contractility and prognosis of postaortic banded rats. SERCA1: SERCA1-TG mice are hyperkinetic. Adeno/SERCA1 has been used to support intramuscular delivery of Kv2.1 in normal guinea pig hearts. 6. Phospholamban (PLN). PLN-TG mice are hypokinetic, whereas knockout mice are hyperkinetic. Adeno and AAV/mutant PLN suppress cardiomyopathic hamster heart failure development. 7. FKBP12.6: In vitro Adeno/FKBP12.6-transfected cardiomyocyte increases contractility. Parvalbumin: Adeno/parvalbumin intramuscular delivery enhances normal rat cardiac relaxation. GRK, GPCR kinase; $I_{\mathrm{Ca}, \mathrm{L}}$, L-type calcium channel (dihydropyridine receptor); RyR, ryanodine receptor; JP-2, junctophilin-2. See supplemental reading list (www.jci.org/cgi/content/full/109/7/849/DC1) for detailed references. 
Table 2

Molecular defects linked to malignant arrhythmias in human and mouse models

Gene

Disease

Defects in conduction system lineage

HF-1b

$\mathrm{Nkx2.5}$

Tbx5

AMPK $\gamma 2$ subunit (PRKAG2)

Defects in intercellular coupling

Connexin 43

Defects in ion-channel subunits

KCNQ1/KVLQT1 (LQT1)

KCNH2/HERG (LQT2)

KCNE1/minK (LQT5)

KCNE2/MiRP1 (LQT6)

SCN5A (LQT3)

RyR2

Defect in a cytoplasmic ion-channel accessory protein

KChIP2

Sustained polymorphic VT

AMPK, AMP-activated protein kinase; $\mathrm{SN}$, sinus node; $\mathrm{AV}$, atrioventricular; $\mathrm{VT}$, ventricular tachycar dia; Af, atrial fibrillation; RyR2, ryanodine receptor 2; KChIP2, Kv channel-interacting protein 2. See supplemental reading list (www.jci.org/cgi/content/full/109/7/849/DC1) for detailed references.

Much of this insight has been gleaned from genetically based forms of arrhythmias in clinical and animal model systems. Thus far, several distinct classes of mutations have been shown to be associated with cardiac arrhythmogenesis (Table 2). For instance, the long QT syndrome, an autosomal-dominant disease associated with an increased incidence of cardiac sudden death, is due to mutations in channel proteins that can lead to prolongation of the QT interval and associated action potential heterogeneity that creates an arrhythmogenic substrate. A second class of arrhythmogenic defects was recently uncovered, which relates to abnormalities in cardiac intercellular conduction that is associated with the loss of cardiac connexins (36). Cardiac sudden death and associated tachy- and bradyarrhythmias have now also been linked with defects in cardiac transcription factors that control electrophysiological target genes in ventricular muscle and conduction system lineages $(23,37,38)$. Recent studies have further implicated storage diseases associated with hypertrophic cardiomyopathy as contributing to progressive conduction defects within the conduction system itself (39-41).

Since it is unlikely that somatic mutations account for the onset of cardiac sudden death in acquired forms of arrhythmogenesis, one key question relates to arrhythmogenesis in progressive heart failure. The loss of an epicardial-endocardial gradient of $I_{\text {to }}$ potassium current, one critical component of cardiac repolarization, represents a highly conserved feature of human heart failure (26). The mechanisms that contribute to the loss of the $I_{\text {to }}$ gradient are unclear, as is the relative importance of changes in this specific current as compared with the multitude of other electrophysiological changes in the failing heart. Interestingly, recent studies have now reported that the loss of $I_{\text {to }}$ can independently increase the dispersion of repolarization and confer marked susceptibility to lethal forms of ventricular tachycardia (42). The observations are based on the discovery of a cyto- plasmic accessory protein (KChIP2) that binds to the $\mathrm{Kv} 4.2$ and 4.3 channel proteins and is markedly downregulated early during pressure overload hypertrophy (Figure 1). This negative regulation mimics the reactivation of the fetal gene program, as the KChIP2 gene is silent in the fetal heart and is expressed only in the adult heart. Mice harboring a mutation in $K C h I P 2$ display a complete loss of the $I_{\text {to }}$ current, whereas heterozygotes for the mutant allele display a $50 \%$ decrease in current density, showing that this channel accessory protein quantitatively regulates the $I_{\text {to }}$ current. Homozygous mutants lack the normal $I_{\text {to }}$ current gradient across the ventricular wall, and they show an inducible sustained polymorphic ventricular tachycardia (42).

In the human heart, Kv4.2 and Kv4.3 are uniformly expressed throughout the ventricular myocardium, whereas KChIP2 is expressed in an epicardial-endocardial gradient that matches the endogenous $I_{\text {to }}$ current (43), suggesting that it is limiting for the activation of that current. It will therefore become of interest to determine whether there is a downregulation of KChIP2 expression in the failing human heart that might account for the loss of the $I_{\text {to }}$ gradient in human heart failure. If so, KChIP2 defects may represent a new class of genetic modifiers that can confer risk for both inheritable and acquired forms of ventricular arrhythmias. Since the expression of KChIP2 is downregulated during cardiac remodeling, strategies to maintain $I_{\text {to }}$ by promoting the level of KChIP2 expression in the failing heart may offer a new therapeutic approach for combating the prevalent lethal arrhythmias associated with heart failure. Other channel accessory proteins, regulating other cardiac currents, may be identified through genomic and proteomic strategies and may confer susceptibility to arrhythmogenesis in heart failure. In this regard, it is interesting that the $I_{\mathrm{Ks}}$ channel $\alpha$ subunit, another protein that binds to KCNQ1 through its leucine zipper motif, has been shown to mediate catecholamine sensitivity of cardiac repolarization (44).

\section{Toward new therapeutic pathways}

Over the past few years, a host of new cancer therapies have been brought to the bedside, with a number of promising newcomers in ongoing clinical trials. In large part, these successes reflect our deepening mechanistic understanding of the role of specific molecular checkpoints for important aspects of cancer initiation, promotion, and progression. In terms of novel, biologically targeted therapy early in this century, there is little doubt that cancer rules. However, in a fashion similar to the recent trend of cancer biology, capitalizing on the expanding genomic databases and connecting them with in vivo physiological function holds great promise for both a scientific and a pharmaceutical windfall in heart failure. In short, understanding the failing heart at a molecular level now seems approachable, by breaking down this complex disease into specific endpoints, 
and identifying key pathways and checkpoints for each of the important clinical phenotypes during heart failure progression. New studies documenting the presence of cardiac stem cells (45), novel cardiac gene delivery systems (46-48), and model organisms that will allow a more rapid physiological evaluation of candidate genes (49) offer exciting new directions for the field.

Lewis Thomas, a clear-eyed observer of medical science in general, makes this cogent argument (50):

"I conclude that the greatest potential value of the successful artificial heart is, or ought to be, its power to convince the government as well as the citizenry at large that the nation simply must invest more money in basic biomedical research. We do not really understand the underlying mechanism of cardiomyopathies at all, and we are not much better off at comprehending the biochemical events that disable the heart muscle or its valves in other more common illnesses. But there are clues enough to raise the spirits of people in a good many basic science disciplines, and any number of engrossing questions are at hand awaiting answers."

Note. Interested readers are referred to the supplemental reading list (www.jci.org/cgi/content/full/ 109/7/849/DC1) for detailed references to the data presented in the tables and figures.

1. Hunter, J.J., Grace, A., and Chien, K.R. 1999. Molecular and cellular biology of cardiac hypertrophy and failure. In Molecular basis of cardiovascular disease. K.R. Chien, editor. W.B. Saunders Co. Philadelphia, Pennsylvania, USA. 211-250.

2. Blume-Jensen, P., and Hunter, T. 2001. Oncogenic kinase signalling. Nature. 411:355-365.

3. Chien, K.R. 2000. Genomic circuits and the integrative biology of cardiac diseases. Nature. 407:227-232.

4. Chien, K.R. 1999. Stress pathways and heart failure. Cell. 98:555-558.

5. Yasukawa, H., et al. 2001. Suppressor of cytokine signaling-3 is a biomechanical stress-inducible gene that suppresses gp130-mediated cardiac myocyte hypertrophy and survival pathways. J. Clin. Invest. 108:1459-1467. DOI:10.1172/JCI200113939.

6. Fischer, T.A., et al. 2001. Activation of cardiac c-Jun NH(2)-terminal kinases and p38-mitogen-activated protein kinases with abrupt changes in hemodynamic load. Hypertension. 37:1222-1228.

7. Molkentin, J.D., and Dorn, I.G., II. 2001. Cytoplasmic signaling pathways that regulate cardiac hypertrophy. Annu. Rev. Physiol. 63:391-426.

8. Yamauchi-Takihara, K., and Kishimoto, T. 2000. A novel role for STAT3 in cardiac remodeling. Trends Cardiovasc. Med. 10:298-303.

9. Rothermel, B.A., et al. 2001. Myocyte-enriched calcineurin-interacting protein, MCIP1, inhibits cardiac hypertrophy in vivo. Proc. Natl. Acad. Sci. USA. 98:3328-3333.

10. Yang, J., et al. 2000. Independent signals control expression of the calcineurin inhibitory proteins MCIP1 and MCIP2 in striated muscles. Circ. Res. 87:E61-E68.

11. Bueno, O.F., et al. 2001. The dual-specificity phosphatase MKP-1 limits the cardiac hypertrophic response in vitro and in vivo. Circ. Res. 88:88-96.

12. Rommel, C., et al. 2001. Mediation of IGF-1-induced skeletal myotube hypertrophy by $\mathrm{PI}(3) \mathrm{K} / \mathrm{Akt} / \mathrm{mTOR}$ and $\mathrm{PI}(3) \mathrm{K} / \mathrm{Akt} / \mathrm{GSK} 3$ pathways. Nat. Cell Biol. 3:1009-1013.

13. Bodine, S.C., et al. 2001. Akt/mTOR pathway is a crucial regulator of skeletal muscle hypertrophy and can prevent muscle atrophy in vivo. Nat. Cell Biol. 3:1014-1019.

14. Naga Prasad, S.V., et al. 2000. Gbetagamma-dependent phosphoinositide 3 -kinase activation in hearts with in vivo pressure overload hypertrophy. J. Biol. Chem. 275:4693-4698.

15. Frey, N., McKinsey, T.A., and Olson, E.N. 2000. Decoding calcium signals involved in cardiac growth and function. Nat. Med. 6:1221-1227.

16. Zou, Y., et al. 2001. Calcineurin plays a critical role in the development of pressure overload-induced cardiac hypertrophy. Circulation. 104:97-101.

17. De Windt, L.J., et al. 2001. Targeted inhibition of calcineurin attenuates cardiac hypertrophy in vivo. Proc. Natl. Acad. Sci. USA. 98:3322-3327.

18. Wettschureck, N., et al. 2001. Absence of pressure overload induced myocardial hypertrophy after conditional inactivation of Galphaq/Galpha11 in cardiomyocytes. Nat. Med. 7:1236-1240.
19. Kasahara, H., et al. 2001. Characterization of homo- and heterodimerization of cardiac Csx/Nkx2.5 homeoprotein. J. Biol. Chem. 276:4570-4580.

20. Morin, S., Charron, F., Robitaille, L., and Nemer, M. 2000. GATA-dependent recruitment of MEF2 proteins to target promoters. EMBO J. 19:2046-2055.

21. Passier, R., et al. 2000. CaM kinase signaling induces cardiac hypertrophy and activates the MEF2 transcription factor in vivo. J. Clin. Invest. 105:1395-1406.

22. Hiroi, Y., et al. 2001. Tbx5 associates with Nkx2-5 and synergistically promotes cardiomyocyte differentiation. Nat. Genet. 28:276-280.

23. Bruneau, B.G., et al. 2001. A murine model of Holt-Oram syndrome defines roles of the T-box transcription factor Tbx5 in cardiogenesis and disease. Cell. 106:709-721.

24. Wang, D., et al. 2001. Activation of cardiac gene expression by myocardin, a transcriptional cofactor for serum response factor. Cell. 105:851-862.

25. Furukawa, T., et al. 2001. Specific interaction of the potassium channel beta-subunit minK with the sarcomeric protein T-cap suggests a T-tubulemyofibril linking system. J. Mol. Biol. 313:775-784.

26. Tomaselli, G.F., and Marban, E. 1999. Electrophysiological remodeling in hypertrophy and heart failure. Cardiovasc. Res. 42:270-283.

27. Houser, S.R., Piacentino, V., 3rd, and Weisser, J. 2000. Abnormalities of calcium cycling in the hypertrophied and failing heart. J. Mol. Cell. Cardiol. 32:1595-1607.

28. Bers, D.M. 2002. Cardiac excitation-contraction coupling. Nature. 415:198-205.

29. Schwinger, R.H., et al. 1999. Reduced $\mathrm{Ca}(2+)$-sensitivity of SERCA 2a in failing human myocardium due to reduced serin-16 phospholamban phosphorylation. J. Mol. Cell. Cardiol. 31:479-491.

30. Sande, J.B., et al. 2002. Reduced level of serine(16) phosphorylated phospholamban in the failing rat myocardium: a major contributor to reduced SERCA2 activity. Cardiovasc. Res. 53:382-391.

31. Rockman, H.A., Koch, W.J., and Lefkowitz, R.J. 2002. Seven-transmembrane-spanning receptors and heart function. Nature. 415:206-212.

32. Minamisawa, S., et al. 1999. Chronic phospholamban-sarcoplasmic reticulum calcium ATPase interaction is the critical calcium cycling defect in dilated cardiomyopathy. Cell. 99:313-322.

33. Marx, S.O., et al. 2000. PKA phosphorylation dissociates FKBP12.6 from the calcium release channel (ryanodine receptor): defective regulation in failing hearts. Cell. 101:365-376.

34. Keating, M.T., and Sanguinetti, M.C. 2001. Molecular and cellular mechanisms of cardiac arrhythmias. Cell. 104:569-580.

35. Marban, E. 2002. Cardiac channelopathies. Nature. 415:213-218.

36. Gutstein, D.E., et al. 2001. Conduction slowing and sudden arrhythmic death in mice with cardiac-restricted inactivation of connexin 43. Circ. Res. 88:333-339.

37. Nguyen-Tran, V.T., et al. 2000. A novel genetic pathway for sudden cardiac death via defects in the transition between ventricular and conduction system cell lineages. Cell. 102:671-682.

38. Schott, J.J., et al. 1998. Congenital heart disease caused by mutations in the transcription factor NKX2-5. Science. 281:108-111.

39. Gollob, M.H., et al. 2001. Identification of a gene responsible for familial Wolff-Parkinson-White syndrome. N. Engl. J. Med. 344:1823-1831.

40. Blair, E., et al. 2001. Mutations in the gamma(2) subunit of AMP-activated protein kinase cause familial hypertrophic cardiomyopathy: evidence for the central role of energy compromise in disease pathogenesis. Hum. Mol. Genet. 10:1215-1220.

41. Arad, M., et al. 2002. Constitutively active AMP kinase mutations cause glycogen storage disease mimicking hypertrophic cardiomyopathy. J. Clin. Invest. 109:357-362. DOI:10.1172/JCI200214571.

42. Kuo, H.C., et al. 2001. A defect in the Kv channel-interacting protein 2 (KChIP2) gene leads to a complete loss of I(to) and confers susceptibility to ventricular tachycardia. Cell. 107:801-813.

43. Rosati, B., et al. 2001. Regulation of KChIP2 potassium channel beta subunit gene expression underlies the gradient of transient outward current in canine and human ventricle. J. Physiol. 533:119-125.

44. Marx, S.O., et al. 2002. Requirement of a macromolecular signaling complex for beta adrenergic receptor modulation of the KCNQ1-KCNE1 potassium channel. Science. 295:496-499.

45. Beltrami, A.P., et al. 2001. Evidence that human cardiac myocytes divide after myocardial infarction. N. Engl. J. Med. 344:1750-1757.

46. Hajjar, R.J., del Monte, F., Matsui, T., and Rosenzweig, A. 2000. Prospects for gene therapy for heart failure. Circ. Res. 86:616-621.

47. Davidson, M.J., et al. 2001. Cardiac gene delivery with cardiopulmonary bypass. Circulation. 104:131-133.

48. Ikeda, Y., et al. 2002. Restoration of deficient membrane proteins in the cardiomyopathic hamster by in vivo cardiac gene transfer. Circulation. 105:502-508.

49. Chen, J.N., and Fishman, M.C. 2000. Genetics of heart development. Trends Genet. 16:383-388.

50. Thomas, L. 1995. Late night thoughts on listening to Mabler's ninth symphony. Penguin Books USA. New York, New York, USA. 64-67. 